\title{
Reometria de Torque, Propriedades Mecânicas e Morfologia de Blendas Compatibilizadas de PA6/PEAD
}

\author{
Pankaj Agrawal, Edcleide M. Araújo, Tomás J. A. Mélo \\ Departamento de Engenharia de Materiais, UFCG
}

\begin{abstract}
Resumo: Neste trabalho investigamos o efeito de diferentes compatibilizantes na reometria de torque, propriedades mecânicas e morfologia das blendas de PA6/PEAD. As blendas foram preparadas em uma extrusora dupla-rosca contra-rotativa com filetes totalmente interpenetrantes, a $240{ }^{\circ} \mathrm{C}$ em todas as zonas e velocidade de $50 \mathrm{rpm}$. A composição das blendas foi de 80/20 (\% em peso) para as blendas binárias de PA6/PEAD e de 80/10/10 (\% em peso) para as blendas ternárias de PA6/Compatibilizante/PEAD. Os resultados mostraram que houve um aumento no torque das blendas de PA6/PEAD com a adição dos compatibilizantes. O compatibilizante PEgAA foi o que apresentou maior reatividade com a PA6. Entretanto, foi observada uma redução no torque devido à degradação. Os resultados de propriedades mecânicas mostraram que houve aumento considerável na resistência ao impacto das blendas de PA6/PEAD quando os compatibilizantes foram adicionados. Estes resultados foram corroborados pelos dados de deformação na ruptura sob tração. Os resultados de MEV mostraram que a adição dos compatibilizantes às blendas de PA6/PEAD reduziu significativamente o tamanho médio das partículas de PEAD, e melhorou a adesão entre as fases desses polímeros, resultando principalmente, em um aumento da resistência ao impacto.
\end{abstract}

Palavras-chave: Blendas poliméricas, poliamida 6, polietileno, compatibilizantes.

\section{Torque Rheometry, Mechanical Properties and Morphology of Compatibilized PA6/HDPE Blends}

Abstract: In this paper, an investigation was made of the effect of different compatibilizers on the torque rheometry, mechanical properties, and morphology of Polyamide 6 (PA6)/High Density Polyethylene (HDPE) blends. The compatibilizers used were PEgAA, PEgMA, and EMA-GMA. The blends were prepared in a counter-rotating intermeshing twin screw extruder, at $240{ }^{\circ} \mathrm{C}$ in all zones, and screw speed of $50 \mathrm{rpm}$. The composition of the blends was 80/20 (wt \%) for PA6/HDPE binary blend and 80/10/10 (wt\%) for PA6/Compatibilizer/HDPE blends. The blends were characterized by torque rheometry, mechanical properties, and Scanning Electron Microscopy (SEM). Torque rheometry analysis has shown that there was an increase in the torque of PA6/HDPE blend when the compatibilizers were added. PEgAA compatibilizer was more reactive with PA6 than the other compatibilizers. However, the torque decreased due to degradation. There was a substantial increase in impact strength of PA6/HDPE blend when the compatibilizers were added. These results were corroborated by the strain at break data. SEM results indicated that the addition of compatibilizers to PA6/PE blend has considerably decreased the HDPE average particles size and improved the adhesion between PA6 and HDPE phases, resulting in an increase in impact strength.

Keywords: Polymer blends, polyamide 6, polyethylene, compatibilizers.

\section{Introdução}

A mistura de dois ou mais polímeros (blendas poliméricas) é uma das estratégias de desenvolvimento de novos materiais com propriedades diferentes daquelas dos polímeros puros, além de apresentar baixo custo, quando comparado à síntese de novos polímeros ${ }^{[1]}$. As blendas de Poliamida 6 (PA6) e Polietileno (PE) combinam as propriedades termo-mecânicas e de barreira ao oxigênio da PA6 com a elevada resistência ao impacto, baixo custo e fácil processabilidade do $\mathrm{PE}^{[2,3]}$. Entretanto, assim como a maioria das blendas, as blendas de PA6 e PE são imiscíveis devido à incompatibilidade química entre os componentes, possuindo elevada tensão interfacial, baixa adesão entre as fases, e apresentando um sistema multifásico ${ }^{[4-6]}$. Enquanto a PA6 é composta por grupos polares, o PE é apolar. De acordo com Deanin \& Manion ${ }^{[7]}$ polímeros que são similares na estrutura, ou de uma forma mais geral, similares em polaridade são menos propensos a repelir um ao outro, e mais propensos a formar blendas miscíveis. Polaridades divergentes, geralmente levam à imiscibilidade. As blendas imiscíveis apresentam propriedades que não são adequadas para aplicações industriais, sendo assim, incompatíveis. Para contornar este problema é necessária a adição de um compatibilizante, copolímero em bloco ou enxertado, que pode reagir quimicamente com uma fase e interagir fisicamente com a outra formando um copolímero in situ na interface. Diversos compatibilizantes têm sido utilizados na

Autor para correspondência: Pankaj Agrawal, Programa de Pós-Graduação em Ciência e Engenharia de Materiais, Departamento de Engenharia de Materiais, UFCG, R. Aprígio Veloso 882, Caixa Postal 10034, CEP: 58109-970, Campina Grande, PB, Brasil. E-mail: pankaj@ig.com.br 
compatibilização das blendas de PA6 e PE. Vallim et al. ${ }^{[8]}$ estudaram o efeito compatibilizante do Polietileno de Alta Densidade reciclado (PEpc) em blendas com PA6. As blendas foram caracterizadas por meio de propriedades mecânicas, teste de Molau e Microscopia Eletrônica de Varredura (MEV). Segundo os autores a presença do PEpc provoca um aumento na tensão máxima e no módulo de elasticidade sob tração para todas as composições estudadas em comparação às respectivas blendas preparadas com o PEAD sem ser reciclado. Essa melhora nas propriedades mecânicas foi atribuída à existência de interações fortes na interface das fases presentes na blenda, devido à presença de grupos carbonila no PEpc gerados pela sua degradação, os quais, podem interagir com os grupos amino-terminais da PA6. Para as composições com o PEpc foi observada, por meio de MEV, uma melhor adesão interfacial e uma distribuição mais estreita nos domínios da fase dispersa, evidenciando a maior adesão interfacial. Através do teste de Molau, foi observado um aumento na turbidez das suspensões obtidas a partir das blendas preparadas com PEpc, o que confirmou o resultado obtido por MEV. Os estudos de propriedades mecânicas, MEV, e teste de Molau, evidenciaram a ocorrência de um efeito compatibilizante do PEpc na blenda com PA6. Armat e Moet ${ }^{[9]}$ estudaram a morfologia e propriedades mecânicas das blendas de PEBD/PA6 compatibilizadas com o anidrido maléico funcionalizado com o copolímero em bloco de estireno-(etilieno-co-butileno)-estireno (MA/SEBS). Segundo os autores, o compatibilizante exerce uma dupla função. Por um lado ele reduz a tensão interfacial do sistema, resultando em uma redução do tamanho das partículas da fase dispersa (PE). Por outro lado, o compatibilizante melhora a adesão interfacial através da formação de micro-pontes. Em concentrações de MA/SEBS maiores que $10 \%$, houve um decréscimo considerável na ductilidade das blendas. Wei et al. ${ }^{[10]}$ estudaram a compatibilização reativa de blendas de PA6/PEBD com três tipos de compatibilizantes: PEBD-g-GMA, SEBS-g-GMA e o E-GMA, todos com o metacrilato de glicidila como grupo funcional. Os resultados mostraram que tanto a dispersão das fases como a adesão interfacial, melhorou em todos os sistemas. O menor tamanho de partícula da fase dispersa (PEBD) foi obtido com a presença do SEBS-g-GMA. Os outros dois foram similares na eficiência de compatibilização. Na literatura, existem poucos artigos sobre compatibilização de blendas de PA6/PEAD. O objetivo deste trabalho é investigar o efeito de diferentes compatibilizantes na reometria de torque, propriedades mecânicas e morfologia das blendas de PA6/PEAD.

\section{Experimental}

\section{Materiais}

Poliamida 6 (PA6) C 216 Natural, fornecida pela Rhodia; Polietileno de Alta Densidade (PEAD) JV060U (IF $=7 \mathrm{~g} / 10 \mathrm{~min}$ ), fornecido pela Braskem; Polietileno de Alta Densidade enxertado com 6\% de Ácido Acrílico (PEgAA),
Polybond 1009 (IF = 5 g/10 min) e Polietileno de Alta Densidade enxertado com 1\% de Anidrido Maléico (PEgMA) Polybond 3009 (IF = $5 \mathrm{~g} / 10 \mathrm{~min}$ ) fornecidos pela Crompton; Terpolímero etileno - metil acrilato - metacrilato de glicidila (EMA-GMA), contendo $25 \%$ de éster e $8 \%$ de metacrilato de glicidila (GMA), LOTADER AX 8900 (IF = 6 g/10 min) fornecido pela Arkema.

\section{Métodos}

\section{Secagem dos materiais}

A PA6 e os compatibilizantes PEgAA, e PEgMA foram secos sob vácuo, à temperatura de $80^{\circ} \mathrm{C}$ por 24 horas.

\section{Reometria de torque}

Os ensaios de reometria de torque foram realizados, em um misturador interno RHEOMIX 600, acoplado a um Reômetro de Torque System 90 da Haake - Büchler, operando com rotores do tipo roller, velocidade de rotação de $50 \mathrm{rpm}$ sob temperatura de $240{ }^{\circ} \mathrm{C}$, durante 20 minutos em atmosfera de ar. A massa total dentro da câmara de mistura foi mantida constante em $50 \mathrm{~g}$ para todas as amostras. A composição das blendas foi de 80/20 (\% em peso) para a blenda binária de PA6/PEAD e 80/10/10 (\% em peso) para as blendas ternárias de PA6/Compatibilizante/PEAD.

\section{Preparação das blendas}

As blendas foram preparadas em uma extrusora dupla rosca contra-rotativa com filetes totalmente interpenetrantes. A temperatura utilizada foi de $240{ }^{\circ} \mathrm{C}$ em todas as zonas e a velocidade da roscas foi de $50 \mathrm{rpm}$. A composição das blendas foi 80/20 (\% em peso) para a blenda binária de PA6/PEAD e 80/10/10 (\% em peso) para as blendas ternárias de PA6/Compatibilizante/PEAD. O material obtido foi granulado e seco em estufa sob vácuo a $80^{\circ} \mathrm{C}$ por 24 horas.

\section{Moldagem das amostras}

As amostras para os ensaios de resistência à tração e resistência ao impacto foram moldadas por injeção, utilizandose uma injetora FLUIDMEC, Modelo H3040. A temperatura de moldagem foi de $240{ }^{\circ} \mathrm{C}$. O tempo de molde fechado foi de 20 segundos.

\section{Ensaios mecânicos de tração}

Os ensaios de resistência à tração foram realizados em um equipamento LR 10K da LLOYD Instruments, operando a uma velocidade de deformação de $50 \mathrm{~mm} / \mathrm{min}$, de acordo com a norma ASTM D 638, na temperatura ambiente. Os resultados foram obtidos a partir de uma média de 10 corpos de prova.

\section{Ensaios mecânicos de impacto}

Os ensaios de resistência ao impacto IZOD foram realizados em corpos de prova entalhados, utilizando-se um equipamento do tipo RESIL 5,5 da CEAST e pêndulo de 2,75 J, de acordo com a norma ASTM D 256, na temperatura am- 
biente. Os entalhes de $1,5 \mathrm{~mm}$ foram feitos em um entalhador NOTSCHVIS da CEAST. Os resultados foram obtidos a partir de uma média de 10 corpos de prova.

\section{Microscopia eletrônica de varredura (MEV)}

A análise morfológica foi realizada no ITEP-PE, utilizando-se um Microscópio Eletrônico de Varredura (MEV), Digital SCANNING MICROSCOPE, modelo JEOL JSM 6360. As superfícies de fratura das amostras entalhadas e submetidas ao ensaio de impacto foram recobertas com ouro usando um SPUTTEER COATER SCDO 50 da BAL-TEC. A voltagem utilizada no filamento foi de $8 \mathrm{kV}$. Os resultados foram utilizados na avaliação do efeito dos agentes compatibilizantes na morfologia das blendas de PA6/PE.

\section{Resultados e Discussão}

\section{Reometria de torque}

A Figura 1 mostra as curvas de torque em função do tempo da PA6 e as blendas de PA6/PEAD e PA6/Compatibilizante/ PEAD. Os compatibilizantes foram adicionados à blenda de PA6/PEAD após 5 minutos de operação do misturador, quando a PA6 e o PEAD já haviam fundido. Quando o PEgAA é adicionado à blenda de PA6/PEAD há um aumento substancial no torque. Entretanto, cerca de 5 minutos após a adição do PEgAA, um decréscimo no torque é observado. Segundo Meier-Haack et al..$^{[1]]}$ e Filippi et al. ${ }^{[12]}$, os grupos carboxila do PEgAA reagem com os grupos terminais amina da poliamida 6 , formando o grupo amida, resultando em copolímero in situ na interface. Na reação entre os grupos terminais amina da PA6 e os grupos carboxila do PEgAA ocorre a formação

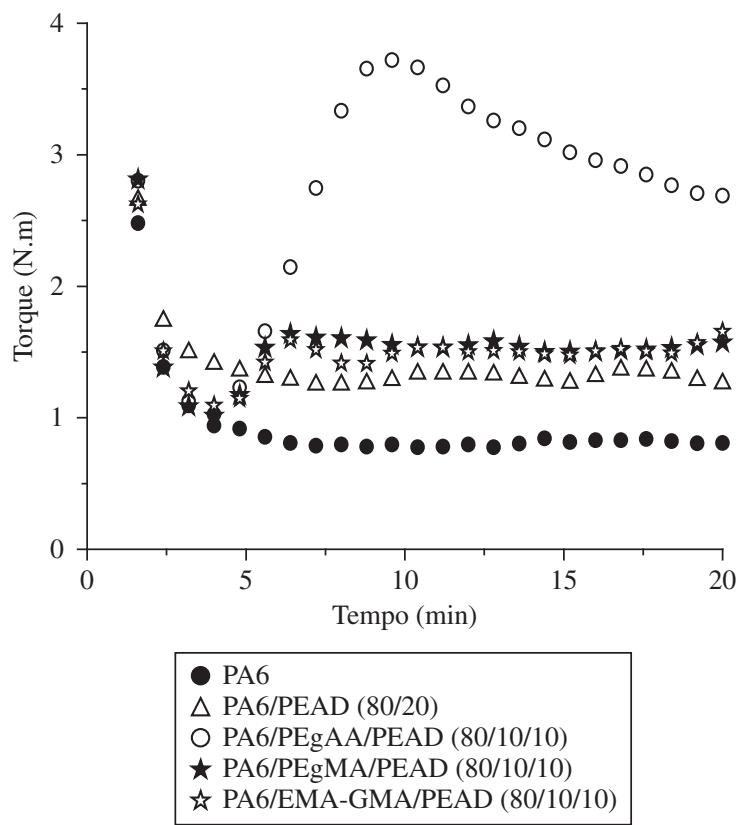

Figura 1. Curvas de torque em função do tempo da PA6 e as blendas de PA6/PEAD e PA6/Compatibilizante/PEAD. de água como subproduto. Esta água provoca a degradação hidrolítica da PA6 por cisão de cadeia, reduzindo o seu peso molecular. Quando o PEgMA é adicionado à blenda de PA6/ PEAD, observa-se um aumento no torque . De acordo com Roeder et al. ${ }^{[13]}$, Jiang et al. ${ }^{[14]}$ e Bassani et al. ${ }^{[15]}$, os grupos anidrido do PEgMA reagem com os grupos terminais amina da poliamida 6 formando o grupo imida e resultando em um copolímero in situ na interface. Assim como acontece com a reação entre os grupos ácidos do PEgAA e os grupos terminais amina da PA6, a reação entre os grupos anidrido do PEgMA e os grupos terminais amina da PA6 também envolve a formação de água como subproduto, que pode levar à degradação das cadeias da PA6 por hidrólise. Entretanto, na Figura 1 observa-se que o torque da blenda PA6/PEgMA/ PEAD apresentou-se constante o que é um indicativo da não ocorrência de degradação. Uma explicação para este comportamento é que o PEgMA contém apenas 1\% de anidrido maléico, o que não seria suficiente para induzir a degradação no mesmo nível que o PEgAA que contém $6 \%$ de ácido acrílico. Quando o EMA-GMA foi adicionado à blenda de PA6/ PEAD, observou-se um aumento no torque. Segundo Koulouri et al. ${ }^{[16]}$, Chiono et al. ${ }^{[17]}$, e Bassani et al..$^{[15]}$, o grupo epóxi presente no GMA pode reagir tanto com os grupos terminais amina quanto com os grupos carboxila da PA6. Comparandose as curvas de torque em função do tempo (Figura 1) das blendas compatibilizadas, observa-se que o torque da blenda de PA6/PEgAA/PEAD foi maior que o das blendas de PA6/PEgMA/PEAD e PA6/EMA-GMA/PEAD, indicando que o PEgAA é mais reativo com a PA6 do que os compatibilizantes PEgMA e EMA-GMA. Não houve diferença significativa entre os torque das blendas de PA6/PEgMA/PEAD e PA6/EMA-GMA/PEAD.

\section{Propriedades mecânicas e morfologia}

A Tabela 1 mostra as propriedades mecânicas da PA6, da blenda binária de PA6/PEAD e das blendas ternárias de PA6/Compatibilizante/PEAD.

Observa-se que a blenda de PA6/EMA-GMA/PEAD foi a que apresentou o menor módulo, o que era esperado, já que o EMA-GMA possui características de um material borrachoso e, portanto, é mais flexível. As blendas de PA6/PEgAA/PEAD e PA6/EMA-GMA/PEAD foram as que apresentaram maior resistência à tração. Já a resistência ao impacto das blendas compatibilizadas foi bem maior do que a da blenda não compatibilizada (PA6/PEAD), o que também é um indicativo de que houve compatibilização reativa, levando a uma redução na diferença de tensão interfacial entre as fases, e conseqüentemente no tamanho médio das partículas da fase dispersa, resultando no aumento da resistência ao impacto. Isto pode ser corroborado por meio da análise da morfologia. A blenda PA6/PEgAA/PEAD foi a que apresentou maior resistência ao impacto. Na Tabela 1 encontram-se também os dados de deformação na ruptura sob tração da PA6, da blenda binária e das blendas ternárias. Observa-se que a deformação na ruptura da blenda binária PA6/PEAD, sem compatibilizante, foi 
Agrawal, P. et al. - Reometria de torque, propriedades mecânicas e morfologia de blendas compatibilizadas de PA6/PEAD

Tabela 1. Propriedades mecânicas da PA6, da blenda binária de PA6/PEAD, e das blendas ternárias de PA6/Compatibilizante/PEAD.

\begin{tabular}{lcccc}
\hline \multicolumn{1}{c}{ Amostra } & E $(\mathbf{G P a})$ & RT $(\mathbf{M P a})$ & RI $(\mathbf{J} / \mathbf{m})$ & $\varepsilon_{\mathbf{r}}(\boldsymbol{\%})$ \\
\hline PA6 & $1,78 \pm 0,10$ & $42,93 \pm 2,20$ & $72,97 \pm 5,38$ & $20,02 \pm 4,54$ \\
PA6/PEAD & $1,48 \pm 0,13$ & $34,92 \pm 1,73$ & $77,52 \pm 8,71$ & $14,26 \pm 6,29$ \\
PA6/PEgAA/PEAD & $1,24 \pm 0,21$ & $39,32 \pm 4,70$ & $242,28 \pm 11,55$ & $177,17 \pm 22,67$ \\
PA6/PEgMA/PEAD & $1,12 \pm 0,10$ & $30,01 \pm 2,80$ & $163,42 \pm 8,32$ & $149,90 \pm 63,12$ \\
PA6/EMA-GMA/PEAD & $0,78 \pm 0,05$ & $38,17 \pm 2,73$ & $170,33 \pm 17,38$ & $199,65 \pm 21,68$ \\
\hline
\end{tabular}

$\mathrm{E}=$ Módulo; RT = Resistência à tração; RI = Resistência ao impacto; e $\varepsilon_{\mathrm{r}}=$ Deformação na ruptura.

menor que a da PA6 indicando que não ocorrem interações entre as fases da blenda corroborando os dados obtidos de resistência ao impacto desta blenda. Por outro lado, a adição dos compatibilizantes à blenda binária PA6/PEAD levou a um aumento substancial da deformação na ruptura, significando que houve um aumento na tenacidade e consequentemente da resistência ao impacto. Portanto, comparando os resultados das propriedades mecânicas das blendas compatibilizadas com os da blenda sem compatibilizante e os da PA6, sugere-se que ocorreu compatibilização reativa entre os grupos funcionais dos compatibilizantes com os grupos funcionais da PA6, conforme já indicado pelo estudo de reometria de torque discutido anteriormente. Em relação à compatibilização da fase dispersa PEAD com os compatibilizantes PEgAA e PEgMA, acredita-se que pode ter ocorrido co-cristalização "parcial" numa única fase uma vez que a base olefínica de ambos compatibilizantes é de polietileno de alta densidade e com baixa concentração de grupos funcionais enxertados, além de possuírem índices de fluidez próximos e consequentemente massas molares próximas ao do PEAD. Entretanto, outros fatores poderão influenciar na co-cristalização, tais como: o grau de ramificações e a distribuição dos grupos funcionais ao longo da cadeia ${ }^{[18]}$. Portanto, para uma melhor compreensão da ocorrência de co-cristalização é necessário maiores investigações por meio de análise térmica (ex. DSC) e/ou difração de raio $\mathrm{X}^{[19]}$. O compatibilizante EMA-GMA, é um terpolímero constituído de etileno (E), metil acrilato (MA) e metacrilato de glicidila (GMA) com 25 e $8 \%$, respectivamente na composição do terpolímero. O percentual de grupos volumosos presentes no EMA-GMA é elevado o que dificultaria a cristalização e, por conseguinte a miscibilidade com o PEAD da fase dispersa. Neste caso o compatibilizante formaria na blenda, preferencialmente uma fase separada do PEAD, porém apresentando reação química dos grupos funcionais epóxi do GMA com a PA6. Por outro lado, a fase de PEAD, teria pouca adesão com a PA6. Este efeito pode ser observado na morfologia da blenda compatibilizada (ver Figura 2d - x1500) onde ocorreu redução no tamanho médio de partículas da fase dispersa de PEAD, e partículas sacadas de PEAD durante o ensaio de impacto, quando comparada com a morfologia da blenda PA6/ PEAD (Figura 2a - x1500). Entretanto, observando os dados das propriedades mecânicas na Tabela 1 , verifica-se que os da blenda PA6/PEAD compatibilizada com EMA-GMA não foram inferiores, exceto no módulo elástico, aos da blenda PA6/ PEAD compatibilizada com PEgMA que apresentou tamanho médio de partícula bem inferior (Figura 2c - x2500). Portanto, comparando os dados na Tabela 1 das blendas de PA6/PEAD com os três tipos de compatibilizantes utilizados, verifica-se que a resposta mecânica nestes casos é mais dependente de mudanças na interface do que do tamanho médio de partículas, e ambos deverão influenciar na atuação dos diferentes mecanismos de tenacificação.

A Figura 2 mostra micrografias das blendas de PA6/PEAD e PA6/Compatibilizante/PEAD. A Figura 2a mostra a micrografia da blenda de PA6/PEAD. Observa-se que esta blenda apresenta um tamanho médio de partículas de PEAD elevado, e não há uma boa adesão entre as fases de PA6 e PEAD, já que diversas partículas de PEAD foram arrancadas da matriz de PA6. Quando os compatibilizantes PEgAA, PEgMA, e EMA-GMA foram adicionados à blenda PA6/PEAD (Figuras 2b, c, e d respectivamente) houve uma redução significativa no tamanho médio das partículas de PEAD, além de uma melhora substancial na adesão entre as fases de PA6 e PEAD.

$\mathrm{Na}$ micrografia da blenda de PA6/PEgAA/PEAD (Figura 2b), observa-se a presença de "pontes" ou "redes" ligando as partículas de PEAD a matriz de PA6. Uma possibilidade para este comportamento é que durante a solidificação ocorre maior contração das partículas dispersas de PEAD levando a um alongamento (deformação plástica) desses ligamentos. Estes ligamentos ("pontes" ou "redes") seriam uma confirmação de que houve a formação de um copolímero in situ na interface ligando a matriz de PA6 com a fase dispersa de PEAD. Tais "pontes" ou "redes" podem ser melhor observadas por meio da Figura 3, que mostra a micrografia da blenda PA6/PEgAA/PEAD com uma ampliação maior.

\section{Conclusões}

Neste trabalho, o efeito de diferentes compatibilizantes na reometria de torque, propriedades mecânicas e morfologia das blendas de PA6/PEAD compatibilizadas foi investigado. Os ensaios de reometria de torque mostraram que houve um aumento no torque das blendas de PA6/PEAD com a adição dos compatibilizantes. A blenda PA6/PEgAA/PEAD foi a que apresentou o maior torque, indicando que o PEgAA foi mais reativo com a PA6 do que os compatibilizantes PEgMA e EMA-GMA. Entretanto, foi observada uma redução no torque devido à degradação. Os resultados de propriedades mecânicas mostraram que houve um aumento considerável na resistência ao impacto das blendas de PA6/PEAD quando os compatibilizantes foram adicionados. Estes resultados fo- 


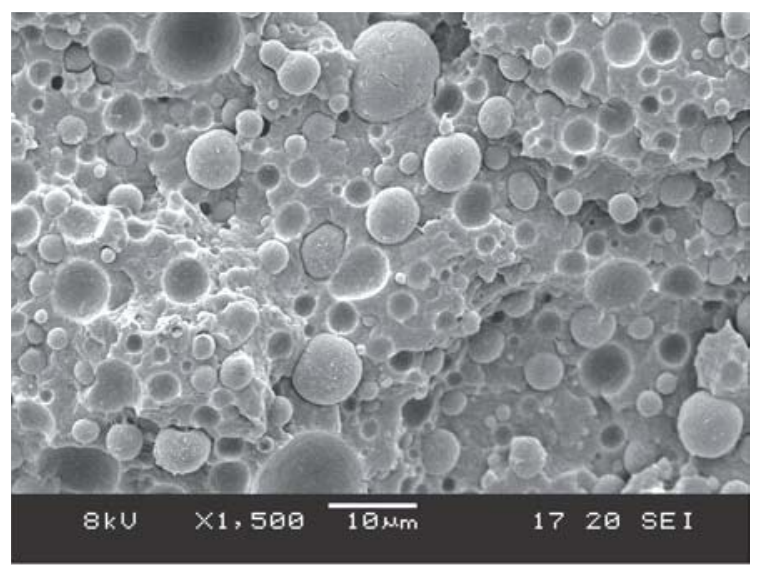

(a)

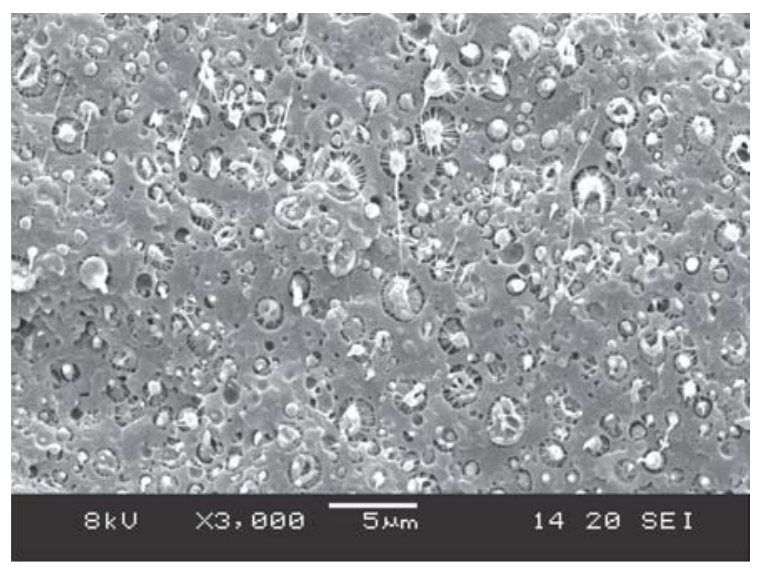

(b)

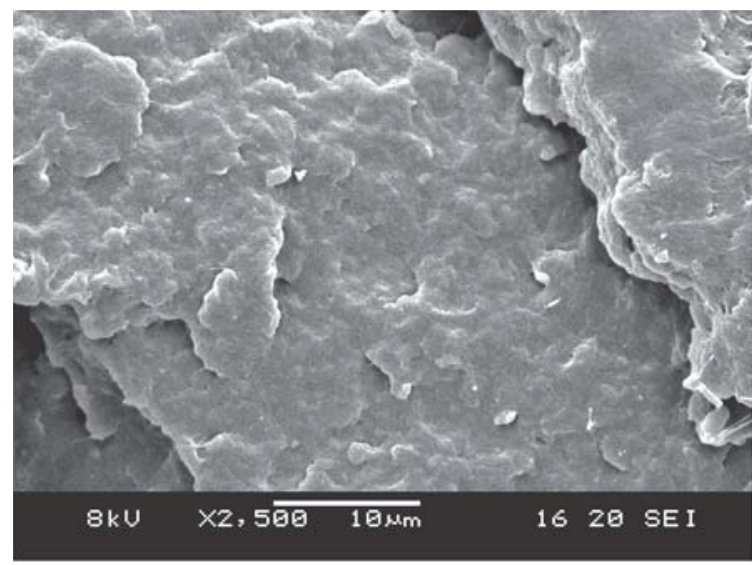

(c)

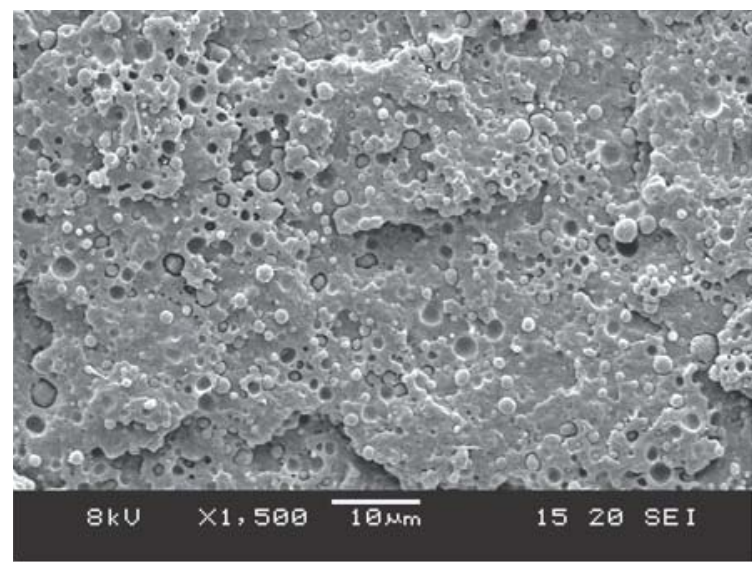

(d)

Figura 2. Micrografias das blendas: a) PA6/PEAD; b) PA6/PEgAA/PEAD; c) PA6/PEgMA/PEAD; e d) PA6/EMA-GMA/PEAD.

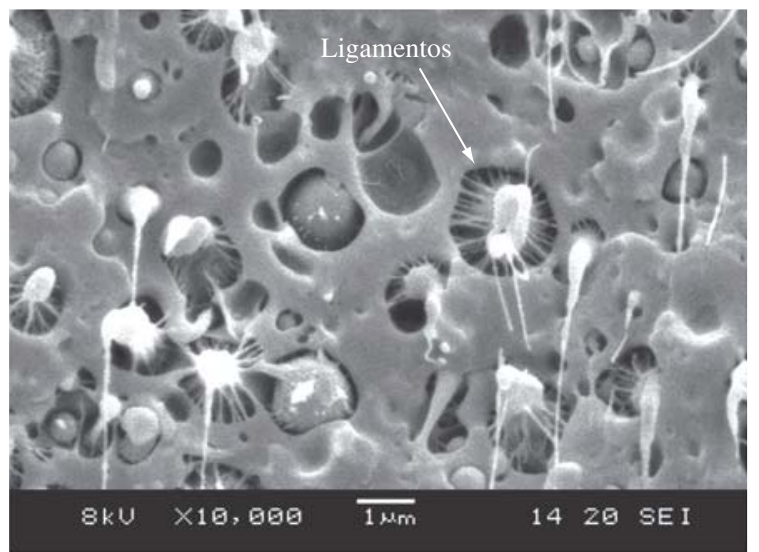

Figura 3. Micrografia da blenda de PA6/PEgAA/PEAD com um aumento de $\times 10000$.

ram corroborados pelos dados de deformação na ruptura sob tração. Os resultados de MEV mostraram que a adição dos compatibilizantes às blendas de PA6/PEAD reduziu significativamente o tamanho médio das partículas de PEAD, e melhorou a adesão entre as fases desses polímeros, resultando em um aumento da resistência ao impacto. Através da análise por MEV também foi observado o aparecimento de "pontes" ou "redes" na blenda de PA6/PEgAA/PEAD (Figura 3), li- gando a fase dispersa de PEAD com a matriz de PA6, o que seria uma confirmação de que houve a formação de um copolímero in situ na interface.

\section{Agradecimentos}

Os autores agradecem à Rhodia pelo fornecimento da PA6, à Crompton pelo fornecimento dos compatibilizantes PEgAA e PEgMA, à Arkema pelo fornecimento do compatibilizante EMA-GMA, à Braskem pelo fornecimento do $\mathrm{PEAD}$, e ao $\mathrm{CNPq}$ pelo apoio financeiro.

\section{Referências Bibliográficas}

1. Utracki, L. A. - "Polymer Blends Handbook", Kluwer Academic Publishers, Netherlands (2002).

2. Lahor, A.; Nithitanakul, M. \& Grady, B. P. - Eur. Polym. J., 40, p.2409 (2004).

3. Valenza, A.; Geuskens, G. \& Spadaro, G. - Eur. Polym. J., 33, p.957 (1997).

4. Tedesco, A.; Barvosa, R. V.; Nachtigall, S.M.B. \& Mauler, R. S. - Polym. Test., 21, p.11 (2002). 
5. Xiaomin, Z.; Gang, L.; Dongmei, W.; Zhihui, Y. \& Jinghua, Y. - Polymer, 39, p.15 (1998).

6. Sacchi, A.; Di Landro, L.; Pegoraro, M. \& Severine, F. Eur. Polym. J., 40, p.1705 (2004).

7. Deanin, R. D. \& Manion, A. M. - "Compatibilization of Polymer Blends", in: Polymer Blends and Alloys, Cap. 1, Gabriel O. Shonaike; George P. Simon (ed.), Marcel Dekker Inc., New York (1999).

8. Vallim, M. R.; Araújo, J. R.; Spinacé, M. A. S.; \& De Paoli, M. A. - "Efeito compatibilizante do PEAD reciclado em blendas com Poliamida 6" in $8^{\circ}$ Congresso Brasileiro de Polímeros, p.1, Águas de Lindóia-SP, novembro (2005).

9. Armat, R. \& Moet, A. - Polymer, 34, p.977 (1993).

10. Wei, Q.; Chionna, D. \& Pracella, M. - Macrom. Chem. Phys., 206, p.777 (2005).

11. Meier-Haack, J.; Valko, M.; Lunkwitz, K. \& Bleha, M. Desalination, 163, p.215 (2004).
12. Fillipi, S.; Chiono, V.; Polacco, G.; Paci, M; Minkova, L. I. \& Magagnini, P. - Macrom. Chem. Phys., 203, p.1512 (2002).

13. Roeder, J.; Oliveira, R. V. B.; Gonçalves, M. C.; Soldi, V. \& Pires, A. T. N. - Polym. Test., 21, p.815 (2002).

14. Jiang, C.; Filippi, S. \& Magagnini, P. - Polymer, 44, p.2411 (2003).

15. Bassani, A.; Hage Jr., E.; Persan, L. A.; Machado, A. V. \& Covas, J. A. - Pol. Cien.Tec., 15, p.176 (2005).

16. Koulouri, E. G.; Georgaki, A. X. \& Kallitsis, J. K. - Polymer, 38, p.4185 (1997)

17. Chiono, V.; Filippi. S.; Yordanov, H.; Minkova, L; \& Magagnini, P. - Polymer, 44, p.2423 (2003).

18. Islam, M. A.; Hussein, I. A. \& Atiqullah, M. - Eur. Polym. J., 43, p.599 (2007).

19. Lee, J. H.; Jeong, Y. G.; Lee, S. C.; Min, B. G. \& Jo, W. H. - Polymer, 43, p.5263 (2002).

Enviado: $21 / 08 / 07$

Reenviado: $17 / 11 / 07$

Aceito: $21 / 11 / 07$ 